BRITIEH LICHEN SOCIETY

\begin{tabular}{lll} 
VOLUME 2 & PART 3 \\
\hline
\end{tabular}

THE

LICHENOLOGIST

PUBLISHED

BY

THE BRITISH LICHEN SOCIETY

Copyright 


\section{OFFICERS, 1964}

\section{Fresident}

A. E. Wade, M.Sc., F.L.S.

Secretary

J. R. Laundon, A.M.A., Department of Botany, British Museum

(Natural History), Cromwell Road, London S.W.7.

Treasurer

J. H. G. Peterken, F.L.S., 73 Forest Drive East, Leytonstone, London E.11.

Editor and Recorder

P. W. James, B.Sc., F.L.S., Department of Botany, British Museum (Natural History), Cromwell Road, London S.W.7.

\section{Assistant Editor}

T. D. V. Swinscow, M.B., B.S., M.Sc., F.L.S., 103 London Road, Knebworth, Herts.

\section{Librarian}

D. C. Smith, D.Phil., Department of Agriculture, The University, Oxford.

\section{Curator}

F. H. Brightman, B.Sc., 2 Red Oak Close, Orpington, Kent.

\section{Conservation Officer}

F. N. Haynes, B.Sc., Department of Biology and Chemistry, Portsmouth College of Technology, Parks Road, Portsmouth, Hants.

Mapping Recorder

M. R. D. Seaward, B.Sc., Lynwode, Vicarage Avenue, Wrawby, Brigg, Lincs.

Elected Members of Council
F. H. Brightman
D. G. Catcheside
F. N. Haynes
J. W. Sheard
C. C. Townsend
C. G. Dobbs

\section{Referees}

Miss U. K. Duncan, M.A., F.L.S., Parkhill, Arbroath, Angus.

P. W. James. Address under "Editor and Recorder."

J. R. Laundon. Address under "Secretary."

S. A. Manning, F.L.S. (Cladonia), 31 The Street, Ringland, Norwich, Norfolk.

F. A. Sowter, F.L.S., Greenholme, Stoughton Lane, Stoughton, Leics. T. D. V. Swinscow (Pyrenocarpous lichens). Address under "Assistant Editor."

A. E. Wade, M.Sc., F.L.S., 71 Marlborough Road, Roath, Cardiff. 